\title{
Sub-Doppler molecular-iodine transitions near the dissociation limit (523-498 nm)
}

\author{
Wang-Yau Cheng, ${ }^{*}$ Lisheng Chen, Tai Hyun Yoon, ${ }^{\dagger}$ John L. Hall, and Jun Ye \\ JILA, National Institute of Standards and Technology and University of Colorado Department of Physics, University of Colorado, \\ Boulder, Colorado 80309-0440
}

Received Novemeber 21, 2001

\begin{abstract}
A widely tunable and high-resolution spectrometer based on a frequency-doubled Ti:sapphire laser was used to explore sub-Doppler transitions of iodine molecules in the wavelength range $523-498 \mathrm{~nm}$. The wavelength dependence of the hyperfine transition linewidth of iodine was mapped out in this region, and the narrowest linewidth was $\sim 4 \mathrm{kHz}$ near $508 \mathrm{~nm}$. The hyperfine-resolved patterns were found to be largely modified toward the dissociation limit. The observed excellent signal-to-noise ratio should lead to high-quality optical frequency standards that are better than those of the popular 532-nm system. (C) 2002 Optical Society of America

OCIS codes: $\quad 020.2930,120.3940,120.4800,300.1030,300.3700$.
\end{abstract}

Hyperfine-resolved optical transitions in molecular iodine $\left(\mathrm{I}_{2}\right)$ often provide stable references for precision spectroscopy. ${ }^{1,2}$ The narrow hyperfine components have also been widely used in laser frequency stabilization, following the lead of an early $\mathrm{He}-\mathrm{Ne} /{ }^{127} \mathrm{I}_{2}$ system by intracavity saturated absorption in a red laser at $633 \mathrm{~nm} .{ }^{3}$ Other systems include $\mathrm{He}-\mathrm{Ne} /{ }^{127} \mathrm{I}_{2}$ at $640,612,576$, and $543 \mathrm{~nm}$ and $\mathrm{Ar} /{ }^{127} \mathrm{I}_{2}$ at $515 \mathrm{~nm}$ as well as the ${ }^{129} \mathrm{I}_{2}$ variants. Most of these systems have wavelengths recommended for realization of the SI Metre. ${ }^{4}$ The $\mathrm{I}_{2}$ lines near $532 \mathrm{~nm}$ are stronger than red transitions, have relatively narrower $(200-300-\mathrm{kHz})$ linewidths, and are readily accessible by frequency-doubled solid-state Nd:YAG lasers. ${ }^{5,6}$ The 532-nm system has proved to be one of the best practical optical frequency standards, with its compact size, reliability, and demonstrated high stability $\left(<5 \times 10^{-14}\right.$ at $\left.1 \mathrm{~s}\right){ }^{7}$ Recent development of wide-bandwidth optical combs shows that such $\mathrm{I}_{2}$-based references can be useful not only for precision length metrology but also for generation of highstability time signals. ${ }^{8}$

To reach higher frequency stability it is useful to explore $\mathrm{I}_{2}$ transitions at wavelengths below $532 \mathrm{~nm}$, where the natural linewidths may decrease at a faster rate than that for the line strengths. In fact, coincidental overlaps between the $\mathrm{I}_{2}$ transitions and corresponding $\mathrm{Ar}^{+}$-ion laser lines at 515 and $501 \mathrm{~nm}$ have already revealed transition linewidths [half-width at half-maximum (HWHM)] as small as $50-100 \mathrm{kHz}$ at these wavelengths., ${ }^{9,10}$ It will be interesting to monitor variations in the transition linewidth when the $\mathrm{I}_{2}$ molecules approach the dissociation limit. Furthermore, a widely tunable laser system also permits systematic studies of rotation-vibration dynamics and hyperfine interactions near the dissociation limit, providing rich information on molecular dynamics. In this Letter we report a widely tunable yet high-resolution and high-sensitivity $\mathrm{I}_{2}$ spectrometer based on a frequency-doubled Ti:sapphire laser. We measured the linewidths of the $\mathrm{I}_{2}$ transitions within the range $523-498 \mathrm{~nm}$; signals were recovered with excellent signal-to-noise ratio $(\mathrm{S} / \mathrm{N})$. Our results indicate that $\mathrm{I}_{2}$ transitions in the wavelength range $532-501 \mathrm{~nm}$ hold great promise for future development of high-quality, portable optical frequency standards, especially in light of the rapid development of all-solid-state Yb:YAG lasers near $1030 \mathrm{~nm} .{ }^{11}$ Further, we show the existence of strong modifications of the hyperfine patterns of $\mathrm{I}_{2}$ near the dissociation limit.

Figure 1 shows part of the experimental setup that implements precision scan and control of the laser frequency. Our single-mode Ti:sapphire laser is constructed to tune from 1080 to $953 \mathrm{~nm}$ and provides $\sim 300 \mathrm{~mW}$ of useful output power at $1030 \mathrm{~nm}$. A small portion of the fundamental power is used for laser frequency stabilization, based on an evacuated, highly stable optical cavity. An external frequency servo based on an acousto-optic modulator (AOM 1) in combination with a piezo transducer- (PZT-) activated laser cavity mirror is used to stabilize the laser frequency onto the cavity resonance, with an in-loop error signal indicating a relative frequency noise below $0.3 \mathrm{~Hz}$. The operational laser linewidth is limited by the vibration noise and drift associated with the cavity. A heterodyne measurement made between the cavity-stabilized Ti:sapphire laser and a $\mathrm{Yb}: \mathrm{YAG}$ laser revealed a beat width of $8 \mathrm{kHz} \mathrm{rms}$, probably limited by the free-running linewidth of the Yb:YAG laser. Frequency scanning of the Ti:sapphire

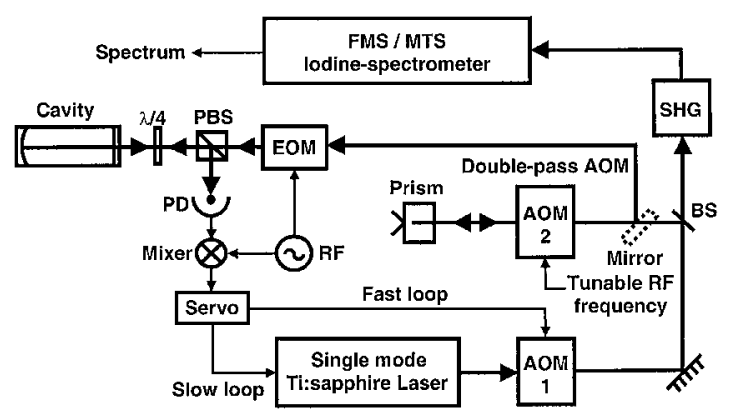

Fig. 1. Part of the experimental setup that controls the laser frequency for the $\mathrm{I}_{2}$ spectrometer: $\mathrm{PD}$, photodiode; EOM, electro-optic modulator; PBS, polarization beam splitter; $\lambda / 4$, quarter-wave plate; BS, beam splitter; other abbreviations defined in the text. 
laser is accomplished with a double-passed acoustooptic modulator (AOM 2) that is located at the cavity input. With a fixed cavity length (aside from drift), changing of the drive frequency of AOM 2 will thus tune the stabilized laser frequency, continuously over a $150-\mathrm{MHz}$ range without losing the cavity lock. Because the cavity's free spectral range (mode spacing) is $244 \mathrm{MHz}(<150 \mathrm{MHz} \times 2)$, this AOM-based laser tuning system allows for continuous laser scan over consecutive cavity modes, basically limited only by the single-mode tuning range of the laser itself.

For second-harmonic generation, a $\mathrm{KNbO}_{3}$ crystal $(3 \mathrm{~mm} \times 5 \mathrm{~mm} \times 3 \mathrm{~mm} ; b$ cut $)$ is placed inside a buildup cavity as the crystal temperature is varied from 172 to $71{ }^{\circ} \mathrm{C}$ to achieve noncritical phase matching at wavelengths that range from 523 to $498 \mathrm{~nm}$. The cavity-output second-harmonic generation power is $82 \mathrm{~mW}$, with a $235-\mathrm{mW}$ mode-matched fundamental power just in front of the doubling cavity. The $\mathrm{I}_{2}$ spectrometer is similar to that described in Ref. 7 but has the added flexibility of using either frequency-modulation spectroscopy (FMS) or modulation-transfer spectroscopy (MTS) for appropriate recovery of a saturated-absorption signal. The high-purity $\mathrm{I}_{2}$ cell was prepared by the Bureau International des Poids et Measures and is $8 \mathrm{~cm}$ long, with a Brewster window at each ends. The $I_{2}$ pressure is controlled by the temperature stabilization of the cell's cold finger.

Figure 2 shows some typical modulation-transfer spectra of $\mathrm{I}_{2}$ transitions from 523 to $500 \mathrm{~nm}$. These spectra were obtained with a vapor pressure of $4.12 \mathrm{~Pa}$. The $\mathrm{I}_{2}$ transitions are identified by the CNRS I ${ }_{2}$ spectral atlas. ${ }^{12}$ However, spectra obtained below $500.53 \mathrm{~nm}$ are out of the range of the $\mathrm{I}_{2}$ atlas. Our $\mathrm{I}_{2}$ spectrometer is found to have a constant noise floor throughout the wavelength range of interest. Strong transitions (with strengths comparable to that of 532-nm lines) are relatively common in the range from 530 to $510 \mathrm{~nm}$, as shown in Fig. 2. Near the dissociation limit $(499.5 \mathrm{~nm}),{ }^{13}$ the line strengths and associated $\mathrm{S} / \mathrm{Ns}$ decrease substantially.

With such a widely tunable spectrometer, a large range of rovibrational quantum numbers can be addressed, permitting a detailed parametric study of transition strengths, hyperfine interactions, and collision physics. For example, Fig. 2(a) shows that the spectrum of $P(54) 38-0$ at $522.474 \mathrm{~nm}$ (even rotational quantum number) follows the usual 15-line hyperfine pattern (1-4-4-1-4-1), whereas the spectrum of $P(61) 43-0$ at $516.505 \mathrm{~nm}$ (odd rotational number) shown in Fig. 2(b) displays the typical 21 hyperfine components (3-4-4-3-4-3). However, as the transition wavelength approaches the predissociation region, for example, line $P(61) 53-0$ at $507.949 \mathrm{~nm}$ [Fig. 2(c)], the hyperfine splitting pattern starts to change. When the wavelength approaches the dissociation limit, as at $500.506 \mathrm{~nm}$, the 15 -line pattern is completely modified [Fig. 2(d)]. Similar observations are made for other lines near $500 \mathrm{~nm}$. Clearly the hyperfine interactions are dramatically influenced by the dissociation effect. Systematic frequency measurement for the corresponding hyperfine constants in this wavelength region is under way in our lab.
The search for the best candidate for an $\mathrm{I}_{2}$-based optical frequency reference requires systematic studies of the linewidth and strength of many transitions in this wavelength region. To obtain the zero-power, zero-pressure linewidth of a particular line we typically vary the $\mathrm{I}_{2}$ cell's pressure from 0.4 to $4 \mathrm{~Pa}$. At each pressure we measure the light-power dependence of the linewidth and determine its zero-power value. We fit the linewidth versus pressure to find the zero-pressure linewidth. Near each wavelength we usually measure and compare a few lines that either have different rovibrational quantum numbers in the excited state or are different hyperfine components within the same transition. Figure 3(a) shows a few pressure-fitted curves. We notice that, even at nearly the same wavelength, natural linewidths of transitions with vastly different rotational quantum numbers can differ by as much as $40 \mathrm{kHz}$ and exhibit quite different collision broadening coefficients. Figure 3(b) displays a number of measured zero-pressure linewidths. For comparison, the 543-nm (Ref. 14) and 532-nm data points are also included. The dashed line $(37-\mathrm{kHz})$ in Fig. 3(b) represents the remediable technical limit to the measured linewidth, including contributions from wave-front curvature and transit time $(\sim 24 \mathrm{kHz})$, laser linewidth (in the green, $\sim 8 \mathrm{kHz})$, recoil $(\sim 5 \mathrm{kHz})$. The natural linewidth can be estimated from deconvolution of the measured Voigt profile. The narrowest natural linewidth that we have found so far is $\sim 43 \mathrm{kHz}$ HWHM for $R(20) 50-0: 65$ at $508.077 \mathrm{~nm}$.

From Fig. 3 we can observe a clear trend of linewidth narrowing with decreasing transition wavelength. However, this tendency is complicated by variations in linewidths among various rotational or hyperfine components when the transitions approach the predissociation region. The initial linewidth narrowing at shorter wavelength may indicate among other interesting effects that the Frank-Condon factor in the transition probability is reduced when the excited state reaches a higher vibration level. As the excited state approaches the dissociation threshold,

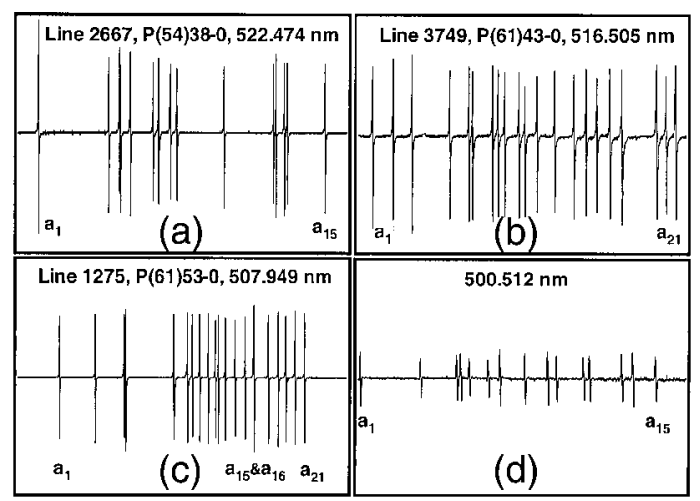

Fig. 2. Typical spectra of molecular $\mathrm{I}_{2}$ hyperfine transitions from $523 \mathrm{~nm}$ to $500 \mathrm{~nm}$. (a), (b), (c) MTS, 273-k Hz modulation frequency; (d) FMS, 6- $\mathrm{MHz}$ modulation frequency. The quoted ${ }^{12}$ line strengths are (a) 80, (b) 79, (c) 55. The time constant is $5 \mathrm{~ms}$ for (a)-(c) and $100 \mathrm{~ms}$ for (d). Length of $\mathrm{I}_{2}$ cell, $8 \mathrm{~cm}$; vapor pressure, 4.12 Pa. 

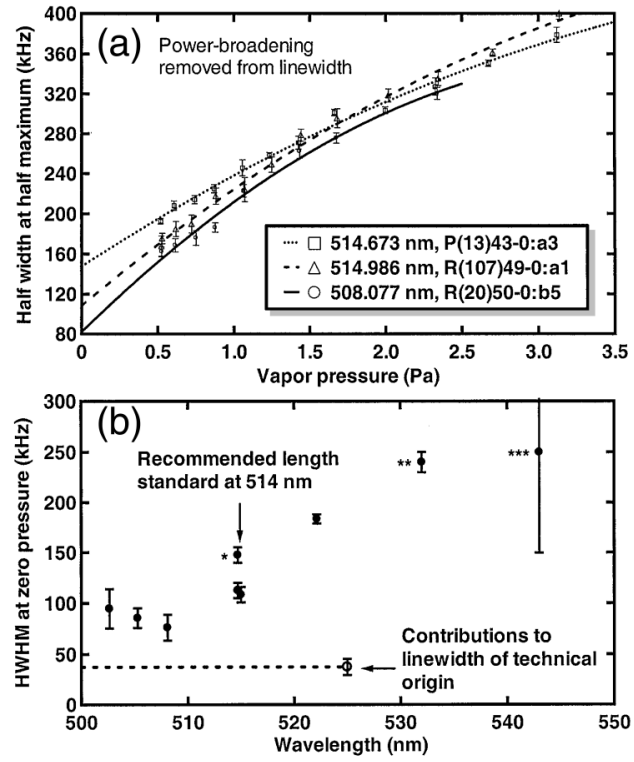

Fig. 3. (a) Three typical curves of zero-power transition linewidth (HWHM) versus pressure. (b) Wavelength dependency of linewidth: *, wavelength standard $P(13) 43-0$, $a 3$ at $514.673 \mathrm{~nm}^{4}$; $* *$, measured by Nd:YAG laser; $* * *$, measured by $\mathrm{He}-\mathrm{Ne}$ laser. ${ }^{14}$

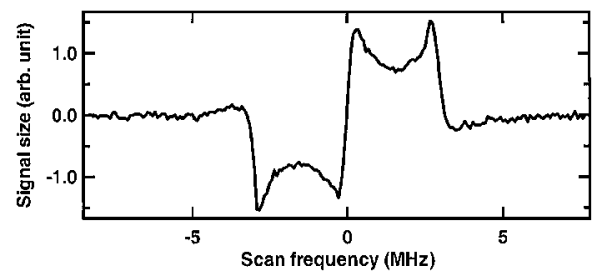

Fig. 4. Typical FMS S/N of a hyperfine transition of $R(86) 57-0$ at $508.060 \mathrm{~nm}$. Laser frequency stabilization with this discrimination slope will lead to a projected (in)stability of $1.6 \times 10^{-13}$ at $1 \mathrm{~s}$, or $1 \times 10^{-14}$ at $1 \mathrm{~s}$ for a 120-cm-long $\mathrm{I}_{2}$ cell.

the limit on lifetime imposed by predissociation will need to be taken into consideration. ${ }^{15}$

To stabilize the frequency of the Ti:sapphire laser we simply feed back the molecular resonance information to the drive frequency of AOM2, which basically suppresses the drift and low-frequency noise from the prestabilization cavity. Currently we use a femtosecond-laser-based wide-bandwidth optical comb to check system performance, which we expect to surpass that of the $\mathrm{Nd}: \mathrm{YAG} / \mathrm{I}_{2}$ system. A preliminary estimate of the system performance at $508 \mathrm{~nm}$ can be made based on the $\mathrm{S} / \mathrm{N}$ of the discrimination curve shown in Fig. 4. The transition is one of the hyperfine components of $R(86) 57-0$ at $508.060 \mathrm{~nm}$, and the signal is recovered with FM spectroscopy with a modulation frequency of $6 \mathrm{MHz}$ and a 1-ms time constant. The cold finger temperature of the $\mathrm{I}_{2}$ cell is $-15{ }^{\circ} \mathrm{C}$, which corresponds to $0.79-\mathrm{Pa}$ vapor pressure. The projected frequency noise of a laser locked to this error signal is $3 \mathrm{kHz}$, corresponding to a fractional noise of $5 \times 10^{-12}$ at $1 \mathrm{~ms}$ or $1.6 \times 10^{-13}$ at $1 \mathrm{~s}$. The best (in)stability reported in an $\mathrm{I}_{2}$-stabilized laser system is $<5 \times 10^{-14}$ at $1 \mathrm{~s}$ with a 120 -cm long $\mathrm{I}_{2}$ cell, also at $-15^{\circ} \mathrm{C} .{ }^{7}$ Therefore the performance of the new system, if it is scaled to the same $\mathrm{I}_{2}$ cell length, would have an (in)stability of $1 \times 10^{-14}$ at $1 \mathrm{~s}$. Placing the $\mathrm{I}_{2}$ cell inside a multipass cavity will further improve system performance.

$\mathrm{I}_{2}$ transitions in the important wavelength region of $523-498 \mathrm{~nm}$ yield a number of highly promising candidates for future optical frequency standards. Using a wide-bandwidth precision optical frequency comb, we are measuring the absolute frequencies and the associated systematic shifts of these new reference lines. We intend to perform detailed studies of molecular dynamics including hyperfine interactions in the region of predissociation as well as to check systematically the formula for the predicted hyperfine-induced predissociation broadening. ${ }^{15}$ Portable systems based on $\mathrm{Yb}$ :YAG laser are under investigation.

We thank L.-S. Ma and M. Notcutt for useful discussions. This research is funded by NASA, Colorado Photonics and Optoelectronics Program, the National Institute of Standards and Technology, and the National Science Foundation. J. Ye's e-mail address is ye@jila.colorado.edu.

*Present address, Department of Physics, National Dong Hwa University, Taiwan, China.

${ }^{\dagger}$ Present address, Korea Research Institute of Standards and Science, Daejeon, Korea.

\section{References}

1. S. Ezekiel and R. Weiss, Phys. Rev. Lett. 20, 91 (1968).

2. M. D. Levenson and A. L. Schawlow, Phys. Rev. A 6, 10 (1972).

3. G. R. Hanes and C. E. Dahlstrom, Appl. Phys. Lett. 14, 362 (1969).

4. T. J. Quinn, Metrologia 30, 523 (1994); and references therein.

5. A. Arie, S. Schiller, E. K. Gustafson, and R. L. Byer, Opt. Lett. 17, 1204 (1992).

6. M. L. Eickhoff and J. L. Hall, IEEE Trans. Instrum. Meas. 44, 155 (1995).

7. J. Ye, L. Robertsson, S. Picard, L.-S. Ma, and J. L. Hall, IEEE Trans. Instrum. Meas. 48, 544 (1999).

8. J. Ye, L.-S. Ma, and J. L. Hall, Phys. Rev. Lett. 87, 2708-01 (2001)

9. Ch. J. Borde, G. Camy, B. Decomps, and J.-P. Descoubes, J. Phys. 42, 1393 (1981).

10. A. N. Goncharov, M. N. Skvortsov, and V. P. Chebotayev, Appl. Phys. B 51, 108 (1990).

11. T. J. Carrig, J. W. Hobbs, C. J. Urbina, G. J. Wagner, C. P. Hale, S. W. Henderson, R. A. Swirbalus, and C. A. Denman, in Advanced Solid-State Lasers, Vol. 34 of OSA Trends in Optics and Photonics Series (Optical Society of America, Washington, D.C., 2000), paper WC12.

12. S. Gerstenkorn and P. Luc, Atlas de Spectre d'Absorption de la Molecule D'Iode 14800$20000 \mathrm{~cm}^{-1}$ (Editions CNRS, Paris, 1985).

13. J. I. Steinfeld, Molecules and Radiation (MIT Press, New York, 1986).

14. W.-Y. Cheng and J.-T. Shy, J. Opt. Soc. Am. B 18, 363 (2001).

15. J. Vigue, M. Broyer, and J. C. Lehmann, J. Phys. 42, 937 (1981). 